\title{
Promoting effect of arachidonic acid supplementation on $N$-methyl- $N$-nitrosourea-induced pancreatic acinar cell hyperplasia in young Lewis rats
}

\author{
KATSUHIKO YOSHIZAWA ${ }^{1}$, NORIHISA UEHARA ${ }^{1}$, AYAKO KIMURA ${ }^{1}$, YUKO EMOTO ${ }^{1,2}$, \\ YUICHI KINOSHITA ${ }^{1,2}$, TAKASHI YURI ${ }^{1}$, HIDEHO TAKADA ${ }^{3}$, TORU MORIGUCHI ${ }^{4}$, \\ TOMOHITO HAMAZAKI ${ }^{5}$ and AIRO TSUBURA ${ }^{1}$
}

\begin{abstract}
${ }^{1}$ Department of Pathology II, Kansai Medical University, Moriguchi, Osaka 570-8506; ${ }^{2}$ Division of Pathology and ${ }^{3}$ Clinical Department of Surgery, Kansai Medical University Takii Hospital, Moriguchi, Osaka 570-8507; ${ }^{4}$ Laboratory of Food and Nutritional Science, Life and Environmental Science, Azabu University, Sagamihara, Kanagawa 252-5201; ${ }^{5}$ Institute of Natural Medicine, University of Toyama, Toyama, Toyama 930-0194, Japan
\end{abstract}

Received July 31, 2012; Accepted October 12, 2012

DOI: $10.3892 / \mathrm{ol} .2012 .1009$

\begin{abstract}
Arachidonic acid (AA) is naturally found in human breast milk. AA, together with docosahexaenoic acid, is commonly added as a functional food ingredient to commercial infant formula worldwide, in accordance with the international standard of Codex Alimentarius. However, few studies have been performed that are concerned with the possible carcinogenic effects of AA supplementation during neonatal life. The effect of dietary AA supplementation in dams, during gestation and lactation, was investigated in $N$-methyl- $N$-nitrosourea (MNU)-induced preneoplastic lesions in the exocrine pancreas of young Lewis rats. Dams were fed either an AA (2.0\% AA) or a basal $(<0.01 \%$ AA) diet. On postnatal day 0 (at birth), male and female pups received a single intraperitoneal injection of either $35 \mathrm{mg} / \mathrm{kg}$ MNU or vehicle. The morphology and proliferating activity of the exocrine pancreas were examined by proliferative cell nuclear antigen immunohistochemistry 7, 14, 21, 28 and/or 60 days post-MNU. Histopathologically, acinar cell hyperplasia $(\mathrm{ACH})$ occurred in the MNU-treated groups 60 days after MNU injection, irrespecitive of whether the rats had been fed an AA diet. Morphometrically, the number and area of $\mathrm{ACH}$ per $1 \mathrm{~mm}^{2}$ in MNU-treated rats increased significantly in the AA diet-fed rats, compared with basal diet-fed rats. The number of proliferative cell nuclear antigenpositive acinar cells in both the normal and hyperplastic areas of MNU-treated rats increased significantly in the AA diet-fed rats. In conclusion, providing dams with an AA-rich
\end{abstract}

Correspondence to: Dr Katsuhiko Yoshizawa, Department of Pathology II, Kansai Medical University, 10-15 Fumizono, Moriguchi, Osaka 570-8506, Japan

E-mail: yoshizak@takii.kmu.ac.jp

Key words: arachidonic acid, fatty acids, $N$-methyl- $N$-nitrosourea, pancreas, acinar cell, hyperplasia, rats diet during gestation and lactation promotes MNU-induced pancreatic $\mathrm{ACH}$ in young Lewis rats.

\section{Introduction}

Arachidonic acid (AA; 20:4n6) is a polyunsaturated fatty acid (PUFA) present in the phospholipids of cell membranes, and is particularly abundant in the retina and brain $(1,2)$. AA present in the human body originates from dietary sources including egg yolk, or is synthesized from linoleic acid (3). AA is naturally occurring in human breast milk. AA, together with docosahexaenoic acid (DHA), is commonly added as a functional food ingredient to commercial infant formula worldwide, in accordance with the international standard of Codex Alimentarius (4). The consensus recommendations by the World Association of Perinatal Medicine and other such societies specify an intake of $0.2-0.5 \%$ DHA of total fatty acids and a level of AA at least equal to that of DHA (5). However, AA has been demonstrated to affect carcinogenesis in certain organs. Skin tumor models in mice, including the 12-O-tetradecanoylphorbol-13-acetate model, have demonstrated an essential role for the AA cascade in skin tumor promotion (6). AA has also promoted the growth of orthotopically transplanted breast cancer cell line tumors (KPL-1 cells) in female athymic BALB/c mice, and of urinary bladder tumors in a medium-term multi-organ rat carcinogenesis study (7).

Pancreatic cancer is the fourth leading cause of cancerrelated mortality in the United States, with an estimated 42,470 new cases and 35,240 mortalities in 2009. This is due to a lack of early disease-specific symptoms and effective screening diagnostics, and poor prognosis at the time of diagnosis (8). Principle risk factors for pancreatic cancer include inherited germline mutations in genes and repeated exposure to $N$-nitroso compounds (through tobacco smoke and during the manufacturing of cured rubber products) or their $N$-nitrosamines precursors (in protein-containing foods dried at high temperatures) (9). Animal models of exocrine pancreatic carcinogenesis have been established in guinea 


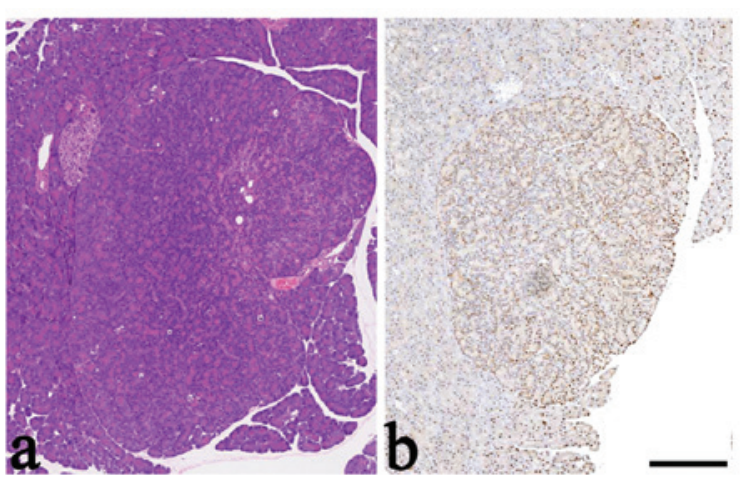

Figure 1. Acinar cell hyperplasia $(\mathrm{ACH})$ in rats treated with $50 \mathrm{mg} / \mathrm{kg}$ $N$-methyl- $N$-nitrosourea (MNU) at birth (day 0 ). Rats were fed a commercial CE-2 diet containing $0.006 \%$ arachidonic acid. (a) Sixteen weeks after MNU treatment. Large $\mathrm{ACH}$ exhibits non-capsulated acidophilic acinar architecture with mild compression to the surrounding tissue. Hematoxylin and eosin (HE) staining was performed. (b) Higher proliferative activity of acinar cells compared to the surrounding acinar cells. Immunohistochemistry was conducted for the proliferating cellular nuclear antigen. Bar=50 $\mu \mathrm{m}$

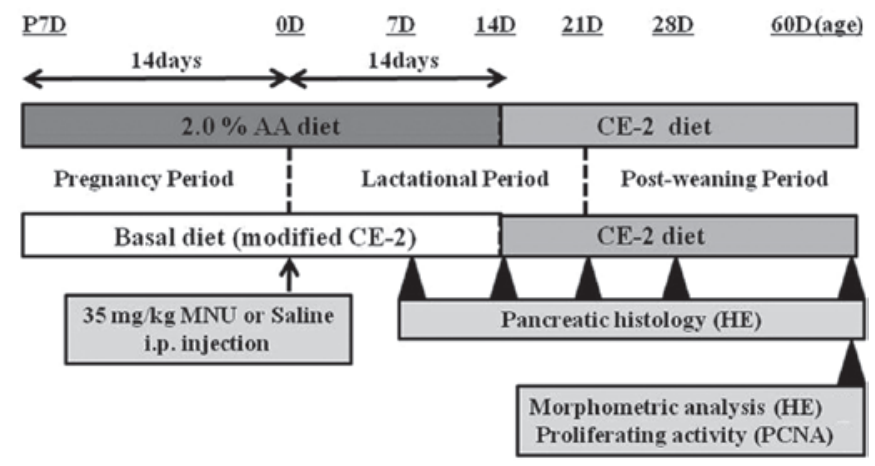

Figure 2. Schema of experimental protocol. AA, arachidonic acid; HE, hematoxylin and eosin; i.p., intraperitoneal; MNU, $N$-methyl- $N$-nitrosourea; PCNA, proliferative cellular nuclear antigen.

pigs, hamsters and/or rodents exposed to several chemicals, including $N$-nitrosamines and azaserine $(10,11)$.

$N$-methyl- $N$-nitrosourea (MNU) is a direct-acting alkylating agent that interacts with DNA. It is toxic and carcinogenic to the immune, hematopoietic, reproductive, mammary, dental, gastrointestinal, nervous and/or sensory systems $(12,13)$. MNU also possesses carcinogenic potency in the pancreas of guinea pigs (14), hamsters (15) and rodents (16). MNU-treated rats develop acinar cell hyperplasia $(\mathrm{ACH})$, which may progress to cancer in certain cases $(16,17)$. These results suggest that increased incidence of $\mathrm{ACH}$ is an early indicator of exocrine pancreatic carcinogenesis (11). In the present study, we aimed to elucidate the promoting effect of prenatal and postnatal dietary AA on MNU-induced exocrine pancreatic carcinogenesis in young Lewis rats.

\section{Materials and methods}

Animal procedures. The study protocol and all animal procedures were approved by the Animal Care and Use Committee of Kansai Medical University, and were in accordance with the guidelines for animal experimentation at Kansai Medical
University. Sixteen female SPF/VAF rats [LEW/CrlCrlj], 10 -weeks-old and one-week pregnant, were purchased from Charles River Japan (Yokohama, Japan). Rats were maintained in specific pathogen-free conditions and had access to water and CE-2-modified diets containing different doses of AA ad libitum. Rats were housed in plastic cages with paper-chip bedding (Paper Clean, SLC, Hamamatsu, Japan) in an airconditioned room at $22 \pm 2{ }^{\circ} \mathrm{C}$ and $60 \pm 10 \%$ relative humidity, with a 12-h light/dark cycle. The illumination intensity inside the cages was less than 60 lux. Offspring were culled to a maximum of 10 per dam and the dams were maintained on their respective diets during the 21-day lactation period. During a post-weaning period of up to 60 days, the offspring were maintained on a CE-2 diet. A total of 116 male and female pups were used in this study. Five to ten rats were sacrificed at each time point, and there was a similar number of males and females in each dietary group.

Chemical and dose formulation. MNU was obtained from Sigma-Aldrich (St. Louis, MO, USA) and was maintained at $-80^{\circ} \mathrm{C}$ in the dark. MNU solution was dissolved in physiological saline containing $0.05 \%$ acetic acid immediately prior to use. MNU (35 mg/kg) or vehicle (physiological saline containing $0.05 \%$ acetic acid) was administered once by intraperitoneal (i.p.) injection. In our preliminary study, pancreatic $\mathrm{ACH}$ developed in 11 of the 14 surviving rats that were treated with $50 \mathrm{mg} / \mathrm{kg} \mathrm{MNU}$ at birth (Fig. 1). However, almost $50 \%$ of these rats did not survive and all surviving female rats developed mammary cancers with severe hematotoxicity. Therefore, $35 \mathrm{mg} / \mathrm{kg}$ MNU was selected as a non-lethal, lower dose that would not cause mammary cancer to occur in the present short-term study.

AA-supplemented diet. AA (purity of $40.4 \%$ by our analysis) was purchased from Cargill Alking Bioengineering (Wuhan) Co, Ltd. (Hubei, China) and purified for use. The diet with $2.0 \mathrm{w} / \mathrm{w} \%$ AA was semi-purified based on the modified CE-2 formulation (CLEA Japan, Inc., Tokyo, Japan). The basal diet consisted of modified CE-2. Gas chromatographic analyses of the fatty acid composition of the diets are displayed in Table I (18). The total fatty acid volumes were 47.20, 86.75 and $126.63 \mu \mathrm{g} / \mathrm{mg}$ of diet for the CE-2 $(0.006 \% \mathrm{w} / \mathrm{w} \mathrm{AA})$, basal $(0.008 \% \mathrm{w} / \mathrm{w} \mathrm{AA})$ and $2.0 \%$ AA diets, respectively. These diets were exposed to $\gamma$-ray (30 Gy) and formulated by CLEA Japan. Food was stored at $4^{\circ} \mathrm{C}$ to prevent lipid oxidation before use.

Experimental procedures. Male and female Lewis rats were exposed to either a basal or an experimental diet (2.0\% AA) from fertilization to sacrifice. At birth ( 0 days of age), rats received an i.p. injection of either vehicle (physiological saline) or $35 \mathrm{mg} / \mathrm{kg}$ MNU (Fig. 2). At 7, 14, 21, 28 and 60 days after MNU or vehicle treatment, rats were anesthetized with isoflurane (Forane; Abbot Japan, Tokyo, Japan) and sacrificed by exsanguination from aortic transection. During the experiment, all pups were observed daily for clinical signs of toxicity, and were weighed at the time of MNU treatment and on the day of sacrifice. The pancreas was immediately removed on sacrifice and complete necropsies were conducted on all animals to check the systemic toxicities induced by 
Table I. Fatty acid composition of diets given to rats.

\begin{tabular}{|c|c|c|c|}
\hline Fatty acid component (wt $\%$ ) & CE-2 & Basal & $2.0 \% \mathrm{AA}$ \\
\hline$\sum$ Saturated & 19.53 & 23.06 & 24.45 \\
\hline$\Sigma$ Monounsaturated & 23.65 & 34.55 & 24.41 \\
\hline$\sum \mathrm{n}-6$ & 41.81 & 31.82 & 42.26 \\
\hline$\sum n-3$ & 7.20 & 5.53 & 3.86 \\
\hline$n-3 / n-6$ & 0.17 & 0.17 & 0.09 \\
\hline AA/DHA & 0.09 & 0.14 & 32.45 \\
\hline \multicolumn{4}{|l|}{ n6 } \\
\hline $18: 2 \mathrm{n} 6$ & 41.56 & 31.58 & 23.73 \\
\hline $18: 3 n 6$ & 0.00 & 0.00 & 0.85 \\
\hline $20: 2 \mathrm{n} 6$ & 0.11 & 0.15 & 0.27 \\
\hline $20: 3 n 6$ & 0.00 & 0.00 & 1.40 \\
\hline $20: 4 n 6$ & 0.14 & 0.10 & 15.90 \\
\hline $22: 2 n 6$ & ND & ND & ND \\
\hline $22: 4 n 6$ & ND & ND & 0.12 \\
\hline $22: 5 n 6$ & ND & ND & ND \\
\hline \multicolumn{4}{|l|}{ n3 } \\
\hline $18: 3 n 3$ & 3.16 & 3.51 & 2.29 \\
\hline $20: 3 n 3$ & 0.00 & 0.00 & 0.02 \\
\hline $20: 5 n 3$ & 2.04 & 1.07 & 0.91 \\
\hline $22: 5 n 3$ & 0.41 & 0.23 & 0.15 \\
\hline $22: 6 n 3$ & 1.59 & 0.73 & 0.49 \\
\hline Total fatty acid $(\mu \mathrm{g} / \mathrm{mg})$ & 47.20 & 86.75 & 126.63 \\
\hline AA\% of total fatty acid & 0.30 & 0.12 & 12.56 \\
\hline
\end{tabular}

AA, arachidonic acid; DHA, docosahexaenoic acid.

AA supplementation. Food consumption and body weight of the dams were measured once per week to estimate the true dosage of AA during the pregnancy and lactation periods.

Tissue fixation and processing. Pancreas tissues were fixed overnight in $10 \%$ neutral buffered formalin, embedded in paraffin, sectioned at a thickness of $4 \mu \mathrm{m}$ and stained with hematoxylin and eosin (HE). Histopathological and morphometrical evaluations were performed by a toxicologic pathologist certified by the Japanese Society of Toxicologic Pathology and/or the International Academy of Toxicologic Pathology (K.Y. and A.T.). Previously defined histopathological terminology and diagnostic criteria were used. $\mathrm{ACH}$ is characterized by non-encapsulate glandular architecture with zymogen granules, exhibiting larger size cytoplasm and nucleus, basophilia, milder compression without higher mitotic index and nuclear polymorphism $(19,20)$. This lesion was distinguished from regenerative hyperplasia that occurs in response to degeneration and necrosis of acinar cells, as regeneration in response to a single episode of severe toxic injury typically results in an almost complete restoration of normal architecture, rather than a focal nodular lesion (19). However, multifocal ACH develops into frank neoplasia (20).

Morphometric analysis of pancreatic ACH. HE-stained pancreas sections obtained from rats sacrificed 60 days after MNU treatment were scanned with a high-resolution digital slide scanner (NanoZoomer 2.0 Digital Pathology; Hamamatsu Photonics, Hamamatsu, Japan) to prepare digital images. The image files were opened with NDP.view software (Hamamatsu Photonics). The total number of ACH lesions were counted on each digital image. The areas of each $\mathrm{ACH}$ and whole pancreas tissue were individually measured using NDP.view software. To further evaluate the quantity and quality of $\mathrm{ACH}$, the average area and number of $\mathrm{ACH}$ per $1 \mathrm{~mm}^{2}$ were calculated.

Proliferating cell nuclear antigen (PCNA) staining and labeling index. Formalin-fixed pancreatic sections from rats (basal and 2.0\% AA diets) sacrificed 60 days after MNU or vehicle treatment were used for proliferative activity analysis. Sequential sections were immunohistochemically evaluated with anti-PCNA monoclonal antibody (clone PC10, dilution 1:100; Leica Biosystems, Newcastle-upon-Tyne,UK). A Labeled Streptavidin Biotin (LSAB) staining kit (Dako, Carpinteria, CA, USA) and antigen retrieval by pressure-cooker heating (Pascal, Dako) were used for immunostaining (21). PCNAstained sections were scanned with a high-resolution digital slide scanner to prepare digital images. The labeling index was calculated from digital images by determining the number of PCNA-positive nuclei of acinar cells per $1 \mathrm{~mm}^{2}$ in normal exocrine pancreatic tissue and $\mathrm{ACH}$, using NDP.view software. 
Table II. Incidence of ACH induced by MNU.

Days after MNU treatment

\begin{tabular}{|c|c|c|c|c|c|c|}
\hline \multirow[b]{2}{*}{ Treatment } & \multirow[b]{2}{*}{ Diet } & \\
\hline & & 7 & 14 & 21 & 28 & 60 \\
\hline \multirow[t]{2}{*}{ Vehicle } & Basal $^{\mathrm{b}}$ & $0(0 / 6)^{\mathrm{d}}$ & $0(0 / 6)$ & $0(0 / 5)$ & $0(0 / 5)$ & $0(0 / 10)$ \\
\hline & $\mathrm{AA}^{\mathrm{c}}$ & $0(0 / 6)$ & $0(0 / 6)$ & $0(0 / 5)$ & $0(0 / 5)$ & $0(0 / 5)$ \\
\hline \multirow[t]{2}{*}{$\mathrm{MNU}^{\mathrm{a}}$} & Basal & $0(0 / 6)$ & $0(0 / 6)$ & $0(0 / 5)$ & $0(0 / 5)$ & $100(5 / 5)$ \\
\hline & AA & $0(0 / 6)$ & $0(0 / 6)$ & $0(0 / 5)$ & $20(1 / 5)^{\mathrm{e}}$ & $75(6 / 8)^{\mathrm{e}}$ \\
\hline
\end{tabular}

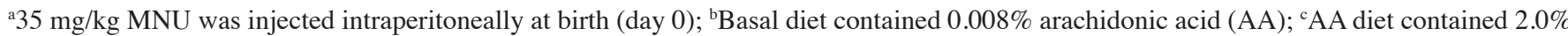
arachidonic acid (AA); 'Percentage of incidence (number of animals with hyperplasia/total number of animals examined) was indicated; ${ }^{\text {e} W i t h o u t ~ s i g n i f i c a n t ~ d i f f e r e n c e ~ b e t w e e n ~ b a s a l ~ d i e t-~ a n d ~ A A-r i c h ~ d i e t-f e d ~ r a t s ~ i n ~ M N U-t r e a t e d ~ g r o u p s . ~ A C H, ~ a c i n a r ~ c e l l ~ h y p e r p l a s i a ; ~ M N U, ~}$ $N$-methyl- $N$-nitrosourea.

Table III. Morphometrical analysis of acinar cell hyperplasia induced by $N$-methyl- $N$-nitrosourea (MNU).

\begin{tabular}{lccr}
\hline Treatment & Diet & Area per hyperplasia $\left(\mathrm{mm}^{2}\right)^{\mathrm{a}}$ & ${\text { Number per } 1 \mathrm{~mm}^{2}}^{2}$ \\
\hline Vehicle & Basal $^{\mathrm{c}}$ & 0 & 0 \\
& AA $^{\mathrm{d}}$ & 0 & 0 \\
MNU $^{\mathrm{b}}$ & Basal & $0.36 \pm 0.18$ & $0.08 \pm 0.02$ \\
& AA & $0.78^{\mathrm{e}} \pm 0.19$ & $0.11^{\mathrm{f}} \pm 0.01$ \\
\hline
\end{tabular}

${ }^{\mathrm{a}}$ Mean \pm standard error; ${ }^{\mathrm{b}} 35 \mathrm{mg} / \mathrm{kg}$ MNU was injected intraperitoneally at birth (day 0); ${ }^{\mathrm{c}}$ The basal diet contained $0.008 \%$ arachidonic acid (AA); ${ }^{\mathrm{d}}$ The AA diet contained $2.0 \%$ arachidonic acid (AA); ${ }^{\mathrm{e}}<0.01$ and ${ }^{\mathrm{f}} \mathrm{P} 0.05$, compared to AA and basal diet groups in MNU-treated rats.

Statistical analysis. All discrete values, expressed as the mean \pm standard error (SE), were analyzed using the two-tailed independent Student's t-test for unpaired samples, after confirming the homogeneity of variances. The incidence of $\mathrm{ACH}$ was analyzed using the $\chi^{2}$ test. The Results include comparisons between basal diet-fed rats and rats fed an AA-supplemented diet, in both the MNU-treated and vehicle-treated groups. $\mathrm{P}<0.05$ was considered to indicate a statistically significant difference.

\section{Results}

General remarks. No fatalities occurred, and no clinical signs or symptoms related to treatment were evident in any of the pups or dams during the experimental period. None of the pups in any of the groups developed mammary tumors. The $2.0 \%$ AA diet did not influence food consumption in dams during the experimental period. During the pregnancy and lactation periods, the AA intake ( $\mathrm{mg} / \mathrm{kg} / \mathrm{day}$ ) of dams was 6.3 and 8.5 in the basal diet group, and 1,477 and 1,876 in the $2.0 \%$ AA group, respectively. The $2.0 \%$ AA diet did not influence body weight gain (the growth rate) in pups or cause weight changes in dams with or without MNU treatment. However, the growth rate of MNU-treated pups was typically lower than that of vehicle-treated pups (data not shown).

Morphological and morphometric analysis. The incidence of proliferative lesions of exocrine pancreatic tissue is demon- strated in Table II. In vehicle-treated rats with or without an AA-rich diet, no proliferative lesions were observed at any time point. In contrast, multifocal ACHs occurred in the basal diet-fed rats 60 days after MNU treatment (100\% incidence). In rats fed an AA-rich diet, multifocal $\mathrm{ACH}$ occurred 28 and 60 days after MNU treatment (20 and 75\% incidence, respectively). There was no significant difference in incidence between MNU-treated rats fed a basal diet and those fed an AA-rich diet. Regardless of diet, the majority of MNU-induced $\mathrm{ACH}$ were characterized by well-differentiated acidophilic acinar cells with cell polarity (Fig. 3). However, among MNU-treated rats, $\mathrm{ACH}$ were larger in rats fed an AA-rich diet, compared with rats fed a basal diet (Fig. 3). In the area surrounding the $\mathrm{ACH}$, there was no evidence of capsulation. The neighboring pancreatic tissue maintained a normal acinar structure with or without mild compression. There was no significant difference in the incidence of ACH between males and females (data not shown). Endocrine pancreatic lesions and acinar cell tumors were not observed in any group at any time point.

The area and number of ACHs per $1 \mathrm{~mm}^{2}$ of exocrine pancreas were determined (Table III). In MNU-treated rats, the area of ACHs was 0.36 in rats fed a basal diet and 0.78 for rats fed an AA-rich diet; thus, the area of $\mathrm{ACH}$ was significantly greater in rats fed an AA-rich diet $(\mathrm{P}<0.01)$. The number of ACHs increased significantly in MNU-treated rats fed an AA-rich diet (0.11), compared with rats fed a basal diet (0.08) $(\mathrm{P}<0.05$; Table III). Thus, the number and area of $\mathrm{ACH}$ were highest in MNU-treated rats fed an AA-rich diet. This 


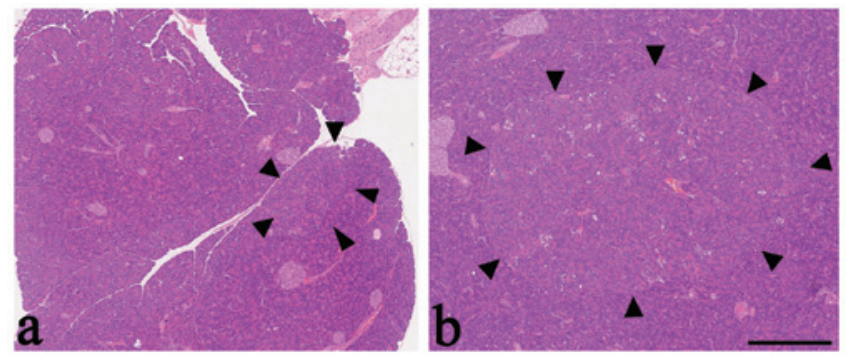

Figure 3. Pancreatic morphology in rats treated with $35 \mathrm{mg} / \mathrm{kg}$ $N$-methyl- $N$-nitrosourea (MNU) at birth (day 0). (a) Small acinar cell hyperplasia (ACH; arrow heads) in a 60-day-old rat that was fed a basal diet containing $0.008 \%$ arachidonic acid (AA). (b) Large ACH (arrow heads) in a 60-day-old rat that was fed a diet containing $2.0 \%$ AA. Hematoxylin and eosin (HE) staining was conducted. Bar $=500 \mu \mathrm{m}$.

$\mathbf{A}$

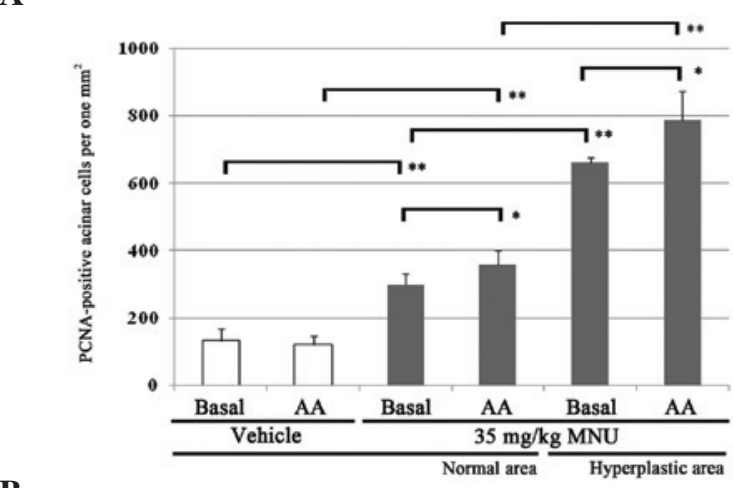

B

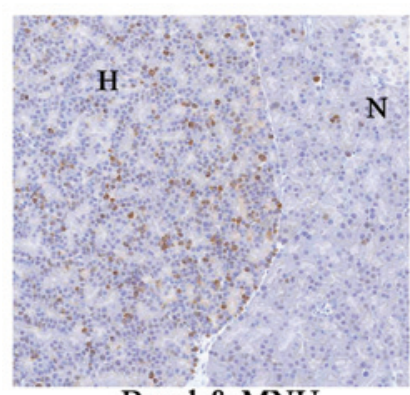

Basal \& MNU

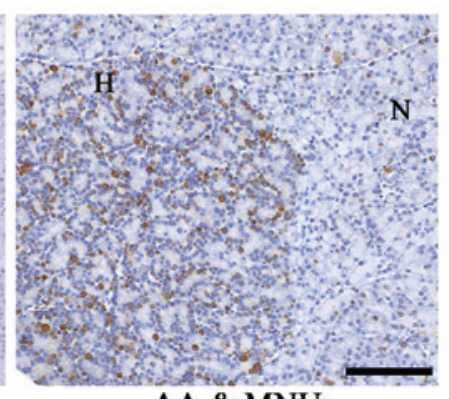

AA \& MNU

Figure 4. Changes in the expression of proliferative cellular nuclear antigen (PCNA)-positive acinar cells in a 60-day-old rat treated with $35 \mathrm{mg} / \mathrm{kg} \mathrm{N} N$-methyl- $N$-nitrosourea (MNU) at birth (day 0). (A) The number of PCNA-positive acinar cells in both the normal and hyperplastic areas increased in MNU-treated rats as compared to vehicle controls. In the MNU-treated rats fed arachidonic acid (AA)-rich food, the number was significantly larger than in the basal food group. Mean \pm standard error, where each bar represents 5-10 rats. $\mathrm{P}<0.05$. (b) Images of immunohistochemistry for PCNA. Proliferative activity of acinar cells was higher in MNU-treated rats fed an AA-rich food than in the basal food group. $\mathrm{H}$, acinar cell hyperplasia $(\mathrm{ACH}) ; \mathrm{N}$, normal area. Bar=100 $\mu \mathrm{m}$.

result was consistent with the morphological characterization (Fig. 3).

Macroscopic examination of other organs. Atrophic changes in the spleen, thymus and testis occurred 7-28 days after MNU treatment, regardless of diet. No macroscopic lesions were detected in any organs, including the pancreas, 60 days after MNU treatment.
PCNA index. In order to compare the cell kinetics of normal acinar areas and $\mathrm{ACH}$ areas among rats fed a basal or AA-rich diet, the PCNA-positive cell number per $1 \mathrm{~mm}^{2}$ of exocrine pancreas was determined. Changes in the number of PCNA-positive acinar cells 60 days after MNU treatment are displayed in Fig. 4. In the basal and AA-rich diet groups, PCNA-positive cells gradually and significantly increased in the normal areas of vehicle- and MNU-treated rat exocrine pancreas, and in the MNU-treated hyperplastic areas. In the normal areas of vehicle-treated rats fed a basal diet or AA-rich diet, the numbers of proliferative acinar cells were 133.2 and 121.0 (no significant difference). In contrast, the number of PCNA-positive cells in normal areas increased significantly in MNU-treated rats fed an AA-rich diet (359.7), compared with rats fed a basal diet (298.7) (Fig. 4A). The number of PCNA-positive cells in ACHs was significantly increased in MNU-treated rats fed an AA-rich diet (786.6), compared with rats fed a basal diet (662.7) (Fig. 4A). Thus, the proliferative activity of acinar cells in normal and $\mathrm{ACH}$ areas was highest in MNU-treated rats fed an AA-rich diet (Fig. 4B).

\section{Discussion}

The main purpose of the present study was to determine whether increased levels of AA during gestation and lactation proportionally enhanced the development of preneoplastic ACHs in the pancreas of MNU-treated rat pups. Pancreatic morphology in rats treated with $35 \mathrm{mg} / \mathrm{kg}$ MNU exhibited small $\mathrm{ACH}$ in rats fed a basal diet and large $\mathrm{ACH}$ in rats fed an AA-rich diet (2.0\% AA). The number and area of ACHs increased in MNU-treated rats fed an AA-rich diet, which was consistent with the morphological characterization. By PCNA immunohistochemistry, the proliferative activity of acinar cells in both the normal and hyperplastic areas was demonstrated to have significantly increased in MNU-treated rats fed an AA-rich diet. These results demonstrated that 2.0\% AA had a strong promoting effect on ACHs, both morphologically and morphometrically. In the MNU rat model of pancreatic carcinogenesis with exposure to $50 \mathrm{mg} / \mathrm{kg}$ MNU at 3 days of age, mancozeb, diethyldithiocarbamate, bayleton, phenobarbital and folithion enhance preneoplastic lesions (acinar cell hyperplasia and dysplasia), carcinoma in situ and/or adenocarcinoma at 24 weeks of age $(16,17,22)$. In our preliminary study, $50 \mathrm{mg} / \mathrm{kg}$ MNU induced mortality and mammary cancers with severe hematotoxicity. Lewis rats are more sensitive than other rat strains to chemical carcinogenesis in the exocrine pancreas. Additionally, rats are most sensitive to the induction of tumors if the chemical is administered during the first several weeks after birth, when the rate of cell division in the pancreas is highest (19). Therefore, as with our experimental protocol, a short-term study (60 days) with $35 \mathrm{mg} / \mathrm{kg}$ MNU as a non-lethal, lower dose that does not cause mammary cancer occurrence, may be extremely useful for testing the promoting, progressing or inhibitory effect of chemical and physical agents on cell proliferation and transformation of rat exocrine pancreas.

High levels of dietary PUFA promote tumor growth in several animal models, including pancreatic cancer models (23). A higher incidence of proliferative exocrine lesions in the pancreas have been observed in F344 rats given 
corn oil in long-term studies $(19,24)$. The promoting effects of unsaturated fats have been attributed to the development of these spontaneously initiated lesions $(19,20)$. In corn oil-treated models, males have a higher incidence and wider distribution of $\mathrm{ACH}$ and tumors than female rats (25), and testosterone is considered to be responsible for the higher incidence of these lesions in males. In the present study, sex differences in the incidence of MNU-induced $\mathrm{ACH}$ were not evident (data not shown), which is likely due to the shorter study period (60 days).

Linoleic acid (LA; 18:2n6) is partly responsible for the promoting effect of dietary polyunsaturated fats on pancreatic carcinogenesis via accelerated prostaglandin synthesis caused by the metabolism of linoleic-derived AA in preneoplastic tissue $(23,26)$. The strongest enhancing effect on the growth of pancreatic (pre)neoplastic lesions in the azaserine rat model and $N$-nitroso bis(2-oxopropyl)amine (BOP) hamster model was obtained with LA-rich diets. In azaserine-induced $\mathrm{ACH}$ (atypical acinar cell foci) in rats fed LA-rich diets, the BrdU-labeling index was significantly increased, compared with rats fed LA-low diets (26). In our study, the proliferative activity of acinar cells in normal and $\mathrm{ACH}$ areas was highest in the MNU-treated rats fed an AA-rich diet, compared with those fed a basal diet. The LA concentration of the AA-rich diet in our study was lower than that of the basal diet (Table I); therefore, LA was not responsible for the promoting effect on $\mathrm{ACH}$ in the present study.

Cholecystokinin (CCK), which is produced and secreted by highly specialized enteroendocrine cells located in the duodenal and jejunal mucosa of the gut, strongly stimulates the secretion of amylase from acinar cells $(19,27)$. Feeding raw soybean flour to rats produces hyperplasia and neoplasia of the exocrine pancreas (28). Additionally, prolonged injection of CCK accelerates the production of atypical acinar cell foci and invasive cancers $(11,19,20)$. Pancreatic growth is considered to be stimulated by interference in the feedback control of CCK by a heat-labile soybean trypsin inhibitor. This effect appears to be independent of any promotion by the high levels of unsaturated fats in the raw soybean flour $(11,20,29)$. The composition of dietary fatty acids influences the CCK secretory response. Short-term exposure of enteroendocrine STC-1 cells to AA promotes CCK secretion (30). Persistent stimulation of the pancreas by CCK induces acinar cell hypersecretion of amylase, followed by hyperplasia, which is capable of promoting chemically induced carcinogenesis of the exocrine pancreas (27,29). Exogenous AA induces amylase secretion in a concentration-dependent manner in the rat acinar cell ex vivo model, suggesting a role for AA as a potential intracellular mediator in the exocrine pancreas (31). In the present study, this information collectively supports our speculation that CCK-related amylase release is involved in the promoting effects of AA on MNU-induced $\mathrm{ACH}$.

Pancreatic cancer is the fourth leading cause of cancer mortality in the United States (8). A previous large population-based, case-control clinical study in San Francisco bay provided evidence that the saturated fatty acids, monounsaturated palmitoleic and oleic fatty acids, and polyunsaturated LA may increase the risk of adenocarcinoma of the exocrine pancreas, whereas gadoleic acid (monounsaturated) and $\omega-3$ fatty acids (polyunsaturated) may decrease this risk (8).
However, no association was observed between pancreatic cancer risk and a dietary intake of $160 \mathrm{mg}$ or more of AA. AA supplementation by healthy adults appears to confer no toxicity or significant safety risk; daily dosages of $1,500 \mathrm{mg}$ for 50 days in the United States and $838 \mathrm{mg}$ for 14 days in Japan have been well-tolerated in clinical studies with no significant side effects $(32,33)$. Previously, AA demonstrated no promoting effects on a rat medium-term multi-organ carcinogenesis model using five carcinogens including MNU (34).

The recommended intake of AA in Japan is $24 \mathrm{mg} / \mathrm{kg} /$ day in adult humans (http:/www.suntory-kenko.com/supplement/ main/433461; in Japanese). The $2.0 \%$ AA diets used in the present study provide an AA dose of $1,477 \mathrm{mg} / \mathrm{kg}$ during pregnancy and $1,876 \mathrm{mg} / \mathrm{kg}$ during lactation, which are 61.6and 78.2-fold higher than the recommended human dose, respectively. Moreover, daily AA intake by Japanese infants via breast milk is approximately $14.3 \mathrm{mg} \mathrm{AA} / \mathrm{kg} /$ day (34). Compared with the amounts of AA tested in the present study, this is approximately 103- and 131-fold higher. Taken together, an AA-enriched diet in the prenatal and postnatal periods is not likely to cause exocrine pancreatic carcinogenesis in humans.

In conclusion, an AA-rich diet in dams during gestation and lactation promotes MNU-induced pancreatic $\mathrm{ACH}$ in young rats. An AA-rich diet induces increased proliferative activity of acinar cells following MNU initiation, likely followed by the development of exocrine pancreatic tumors. Several factors, including AA itself, may affect the increased proliferative activity of the exocrine pancreas. Further studies of the cascade of proliferative action are necessary to understand the detailed mechanisms of the promoting effects of AA on exocrine pancreatic carcinogenesis.

\section{Acknowledgements}

This research was supported in part by Health and Labour Sciences Research Grants (H22-Shokuhin-Ippan-002). The authors thank Ms. T. Akamatsu for her excellent technical assistance, Ms. A. Shudo for manuscript preparation and Dr T. Sasaki (Maruho Co. Ltd.) for her scientific advice.

\section{References}

1. Davis-Bruno K and Tassinari MS: Essential fatty acid supplementation of DHA and ARA and effects on neurodevelopment across animal species: a review of the literature. Birth Defects Res B Dev Reprod Toxicol 92: 240-250, 2011.

2. Uauy R, Hoffman DR, Peirano P, Birch DG and Birch EE: Essential fatty acids in visual and brain development. Lipids 36 : 885-895, 2001.

3. Le HD, Meisel JA, de Meijer VE, Gura KM and Puder M: The essentiality of arachidonic acid and docosahexaenoic acid Prostaglandins Leukot Essent Fatty Acids 81: 165-170, 2009.

4. Codex Alimentarius Commission, Joint FAO/WHO Food Standards Programme: Report of the 28th Session of the Codex Committee on Nutrition and Foods for Special Dietary Uses, 2007.

5. Hoffman DR, Boettcher JA and Diersen-Schade DA: Toward optimizing vision and cognition in term infants by dietary docosahexaenoic and arachidonic acid supplementation: a review of randomized controlled trials. Prostaglandins Leukot Essent Fatty Acids 81: 151-158, 2009.

6. Anonymous: Final report on the safety assessment of arachidonic acid. Int J Toxicol 12: 481-559, 1993

7. Hamazaki T: Reports on "Research on the toxicity of arachidonic acid supplementation". The Health and Labor Sciences Research Grants, Japan (H22-Shokuhin-Ippan-002), 2012 (In Japanese). 
8. Gong Z, Holly EA, Wang F, Chan JM and Bracci PM: Intake of fatty acids and antioxidants and pancreatic cancer in a large population-based case-control study in the San Francisco bay area. Int J Cancer 127: 1893-1904, 2010.

9. Risch HA: Etiology of pancreatic cancer, with a hypothesis concerning the role of $\mathrm{N}$-nitroso compounds and excess gastric acidity. J Natl Cancer Inst 95: 948-960, 2003.

10. Rao MS: Animal models of exocrine pancreatic carcinogenesis. Cancer Metastasis Rev 6: 665-676, 1987.

11. Woutersen RA, van Garderen-Hoetmer A, Lamers CB and Scherer E: Early indicators of exocrine pancreas carcinogenesis produced by non-genotoxic agents. Mutat Res 248: 291-302, 1991.

12. Kimura A, YoshizawaK, Sasaki T,UeharaN,Kinoshita Y,MikiH, Yuri T, Uchida T and Tsubura A: N-methyl-N-nitrosoureainduced changes in epithelial rests of Malassez and the development of odontomas in rats. Exp Ther Med 4: 15-20, 2012

13. Tsubura A, Lai YC, Miki H, Sasaki T, Uehara N, Yuri T and Yoshizawa K: Animal models of N-methyl-N-nitrosoureainduced mammary cancer and retinal degeneration with special emphasis on therapeutic trials. In Vivo 25: 11-22, 2011.

14. Reddy JK and Rao MS: Pancreatic adenocarcinoma in inbred guinea pigs induced by $\mathrm{N}$-methyl-N-nitrosourea. Cancer Res 35 : 2269-2277, 1975

15. Furukawa F,Sato H,Imaida K, Toyoda K,Imazawa T, Takahashi M and Hayashi Y: Induction of pancreatic tumors in male Syrian golden hamsters by intraperitoneal $N$-methyl- $N$-nitrosourea injection. Pancreas 7: 153-158, 1992.

16. Monis B and Valentich MA: Promoting effects of mancozeb on pancreas of nitrosomethylurea-treated rats. Carcinogenesis 14 929-933, 1993.

17. Valentich MA, Eynard AR, Barotto NN, Diaz MP and Bongiovanni GA: Effect of the co-administration of phenobarbital, quercetin and mancozeb on nitrosomethylurea-induced pancreatic tumors in rats. Food Chem Toxicol 44: 2101-2105, 2006.

18. Harauma A and Moriguchi T: Dietary n-3 fatty acid deficiency in mice enhances anxiety induced by chronic mild stress. Lipids 46 : 409-416, 2011.

19. Eustis SL, Boorman GA and Hayashi Y: Exocrine pancreas. In: Pathology of the Fischer Rat: Reference and Atlas. Boorman GA, Montgomery CA Jr and MacKenzie WF (eds). Academic Press, San Diego, pp95-108, 1990.

20. Longnecker DS and Millar PM: Tumors of the pancreas. In: Pathology of Tumors in Laboratory Animals, Vol 1, Tumours of the Rat. Turusov VS and Mohr U (eds). IARC Scientific Publications No. 99, International Agency for Research in Cancer, Lyon, pp241-257, 1990.

21. Yoshizawa K, Sasaki T, Kuro M, Miki H, Kimura A, Uehara N, Yuri T and Tsubura A: Corneal damage induced in adult mice by a single intraperitoneal injection of N-ethyl-N-nitrosourea. In Vivo 25: 609-616, 2011.
22. Barotto NN, López CB, Eynard AR, Fernández Zapico ME, Valentich MA: Quercetin enhances pretumorous lesions in the NMU model of rat pancreatic carcinogenesis. Cancer Lett 129: $1-6,1998$

23. Appel MJ, van Garderen-Hoetmer A and Woutersen RA: Effects of dietary linoleic acid on pancreatic carcinogenesis in rats and hamsters. Cancer Res 54: 2113-2120, 1994.

24. Boorman GA: Proliferative exocrine pancreatic lesions in rats. The effect of sample size on the incidence of lesions. Toxicol Pathol 15: 451-456, 1987.

25. Dominick MA, Bobrowski WF and Metz AL: Proliferative exocrine pancreatic lesions in aged Wistar rats. Toxicol Pathol 18: 423-426, 1990

26. Appel MJ and Woutersen RA: Modulation of growth and cell turnover of preneoplastic lesions and of prostaglandin levels in rat pancreas by dietary fish oil. Carcinogenesis 15: 2107-2112, 1994.

27. Yoshizawa K, Marsh T, Foley JF, Cai B, Peddada S, Walker NJ and Nyska A: Mechanisms of exocrine pancreatic toxicity induced by oral treatment with 2,3,7,8-tetrachlorodibenzo- $p$-dioxin in female Harlan Sprague-Dawley rats. Toxicol Sci 85: 594-606, 2005.

28. McGuiness EE, Morgan RGH and Wormsley KG: Effects of soybean flour on the pancreas of rats. Environ Health Perspect 56: 205-212, 1984

29. Haschek WM, Rousseaux CG and Wallig MA (eds): Pancreas, Section I, Exocrine pancreas. In: Fundamentals of Toxicologic Pathology. 2nd edition. Academic Press, San Diego, pp237-251, 2010.

30. Hand KV, Bruen CM, O'Halloran F, Giblin L and Green BD: Acute and chronic effects of dietary fatty acids on cholecystokinin expression, storage and secretion in enteroendocrine STC-1 cells. Mol Nutr Food Res 54: S93-S103, 2010.

31. Hou W, Arita Y and Morisset J: Endogenous arachidonic acid release and pancreatic amylase secretion. Pancreas 14: 301-308, 1997.

32. Kusumoto A, Ishikura Y, Kawashima H, Kiso Y, Takai S and Miyazaki M: Effects of arachidonate-enriched triacylglycerol supplementation on serum fatty acids and platelet aggregation in healthy male subjects with a fish diet. Br J Nutr 98: 626-635, 2007.

33. Nelson GJ, Schmidt PC, Bartolini G, Kelley DS and Kyle D: The effect of dietary arachidonic acid on platelet function, platelet fatty acid composition, and blood coagulation in humans. Lipids 32: 421-425, 1997.

34. Imai N, Kawabe M, Tamano S, Doi Y, Nakashima H, Suguro M, Numano T, Hara T, Hagiwara A, Furukawa F, Kaneda Y, et al: Arachidonate-enriched triglyceride oil does not promote tumor development in a rat medium-term multi-organ carcinogenesis model. Food Chem Toxicol 50: 2780-2791, 2012. 